\title{
Overcoming the Japanese "Vaccine Gap": An Analysis of Medical Leaders' Witness
}

Miori Tomisaka $^{1^{*}}$, Tomohiko Makino ${ }^{2}$ and Eiji Marui ${ }^{1}$

${ }^{1}$ Department of Public Health, Juntendo University School of Medicine, Japan

${ }^{2}$ Infectious Diseases Surveillance Center, National Institute of Infectious Diseases, Japan

"Corresponding Author: Miori Tomisaka, Department of Public Health, Juntendo University School of Medicine, 2-1-1, Hongo, Bunkyo-ku, Tokyo, Japan, Tel: +81.3.5802.1049; Fax: +81.3.3814.0305; E-mail: miorit@gmail.com

Received date: 25 November 2014; Accepted date: 16 January 2015; Published date: 20 January 2015

Copyright: (c) 2015 Tomisaka M, et al. This is an open-access article distributed under the terms of the Creative Commons Attribution License, which permits unrestricted use, distribution, and reproduction in any medium, provided the original author and source are credited.

\begin{abstract}
Objective: To evaluate the structural challenges in the Japanese national immunization program (NIP) that has caused the "Vaccine Gap".

Methods: Medical opinion leaders out of four stakeholder categories (political, policy, practice, and public) were selected and interviewed. Their observations were analyzed and applied to a case of policy change of replacing oral polio vaccine with inactivated vaccine.

Results: Three issues were identified as the cause of the vaccine gap. First was the lack of evidence-based policy making, derived from the lack of public health human resources and shortage of cost effectiveness studies, as well as the weak surveillance and risk management of adverse events related to immunization. Second was the inappropriate public perception and education for public that can be improved by the strong communication strategy. Third was the weak vaccine development and manufacturing capacities. The case study pointed out that the interaction of public perception and political dynamism at local level can successfully plug the scientific evidence into the national policy.
\end{abstract}

Conclusions: The Japanese vaccine gap can be improved by strengthening public health infrastructures. Political leadership can facilitate the national policy change.

Keywords: Vaccine gap; Adverse events following immunization; Japan; Evidence based policy; Polio; National security

\begin{abstract}
Abbreviations
NIP: National Immunization Program; MMR: Measles-MumpsRubella; MHLW: Ministry of Health, Labour and Welfare; PCV: Pneumococcal Conjugate Vaccine; Hib: Haemophilus influenzae Type b; HPV: Human Papillomavirus; PPV: Pneumococcal Polysaccharide Vaccine; AEFI: Adverse Events Following Immunization; CRPS: Complex Regional Pain Syndrome; PMDA: Pharmaceutical and Medical Device Agency; OPV: Oral Polio Vaccine; IPV: Inactivated Polio Vaccine; ACIP: Advisory Committee on Immunization Practice; NICE: National Institute for Health and Care Excellence; HTA: Health Technology Assessment; VAPP: Vaccine Associated Paralytic Poliomyelitis; DPT: Diphtheria Pertussis Tetanus; VPD: Vaccine Preventable Diseases; NITAG: National Immunization Technical Advisory Group
\end{abstract}

\section{Introduction}

The Japanese National Immunization Program (NIP) has evolved in response to social and public needs since its establishment in 1948. Despite the potential capacity of Japanese researchers and manufacturers for vaccine development, the Japanese NIP has been regarded as delayed in comparison to the programs in other developed nations. The absence of a long term vision in Japanese vaccination policy and the slow processes for approving new vaccines have been key issues in the vaccines debate in Japan [1]. This situation has been negatively termed as the "Vaccine Gap" in Japan [2]. The major cause has been considered to be the excess expectations placed on vaccine safety in Japan [3].

Table 1 lists the major historic events that have impacted the changes in the Japanese NIP. A series of aseptic meningitis cases following Measles-Mumps-Rubella trivalent vaccine (MMR) not only led to ceasing the use of MMR but also to the amendment of the Japanese Immunization Law in such a way as to deny mandatory vaccination in 1994 [1,3]. Since then, the vaccination of children has come to be no longer regarded as a duty of parents. Routine vaccinations financially subsidized by the NIP had little chance of being expanded over these years, only widening the vaccine gap. The emerging increase of influenza among the elderly and the potential threat of a pandemic of H1N1 influenza altered the public climate from an anti-vaccine attitude to a pro-vaccine one, resulting in the creation of two categories of NIP (group 1 for herd immunity and group 2 for individual protection). The pandemic of influenza in 2009 led the government to develop contingent vaccination plans for national security. Upon reflection on the vaccine shortage experienced in the pandemic influenza in 2009, the Ministry of Health, Labour and Welfare (MHLW) recognized a national need for vaccines and production capacities, and started its process to fill the "Vaccine Gap" by including vaccines in its routine schedule [4]. In 2010, MHLW started a facilitation program with temporary budgetary support for 
immunization of pediatric Pneumococcal Conjugate Vaccine (PCV), pediatric Haemophilus influenzae Type b (Hib) vaccine, and Human Papillomavirus (HPV) vaccine. Although four fatal cases following the use of these vaccines [5] led to the temporary suspension of the promotion program in March 2011, these three vaccines were eventually included in the NIP in April 2013 [4]. Varicella and adult Pneumococcal Polysaccharide Vaccine (PPV) were also included into the routine immunization since October 2014. However, public hypersensitivity still impacts NIP. Safety concerns about oral polio vaccination motivated both political and non-governmental groups to push for switching to the use of inactivated polio vaccine. However, the NIP is still vulnerable to suspension as the result of the occurrence of Adverse Events Following Immunization (AEFI) such as encephalitis related to Japanese encephalitis vaccination, and Complex Regional Pain Syndrome (CRPS) following HPV vaccination. A single event of AEFI has driven the public into a hyper-sensitive attitude toward vaccine safety, resulting in massive vaccine hesitancy. Chronic pain cases following HPV vaccination halted national recommendation of HPV on June 14 2013, right after its inclusion into the routine schedule.

\begin{tabular}{|c|c|c|c|c|}
\hline Year & Mission of vaccination & Legal status of vaccination & Social background & Triggering event \\
\hline 1948 & $\begin{array}{l}\text { Public health promotion and } \\
\text { social security }\end{array}$ & $\begin{array}{l}\text { Mandatory vaccination with penalties for } \\
\text { incompliance }\end{array}$ & $\begin{array}{l}\text { Recovery from the defeat in the } \\
\text { WW second }\end{array}$ & US influence through UN/GHQ \\
\hline 1994 & $\begin{array}{l}\text { Autonomy preempts social } \\
\text { security }\end{array}$ & Routine vaccination with subsidies & $\begin{array}{l}\text { Hypersensitivity in safety } \\
\text { concern }\end{array}$ & $\begin{array}{l}\text { MMR meningitis, anti-vaccine } \\
\text { movement for incomplete influenza } \\
\text { vaccine }\end{array}$ \\
\hline 2001 & Social and individual protection & $\begin{array}{l}\text { Classification of group } 1 \text { (maintain herd } \\
\text { immunity) and } 2 \text { (individual protection) }\end{array}$ & $\begin{array}{l}\text { Re-recognition of burden of } \\
\text { VPD }\end{array}$ & $\begin{array}{l}\text { re-emerging of influenza burden on } \\
\text { elderly }\end{array}$ \\
\hline 2012 & National security & $\begin{array}{l}\text { Emergency preparedness of pandemic } \\
\text { and bioterrorism }\end{array}$ & $\begin{array}{l}\text { Protection of essential social } \\
\text { employees }\end{array}$ & H1N1 pandemic influenza in 2009 \\
\hline 2013 & Evidence based vaccine policy & National panel for vaccine evaluation & $\begin{array}{l}\text { Development and importation } \\
\text { of new vaccines }\end{array}$ & HPV, Hib, PCV subsidies \\
\hline
\end{tabular}

Table 1: Key events and influences upon the social mission of vaccination.

It is generally said that the verdict against an officer of MHLW in a lawsuit regarding HIV-contaminated blood products has made MHLW officials very conservative about introducing new pharmaceuticals and biologics [6]. MHLW has also been criticized for its intense favoritism toward domestic manufacturers and the potential cozy relationships. In the past, MHLW has been very reluctant to take steps towards new policy, and its main policy measures have been to offer subsidies to patients [6], but not a national-level compensation scheme for AEFI. Adding new vaccines to the routine schedule has been very hard to introduce because AEFI related to routine vaccinations require a national level compensation scheme, while AEFI from voluntary vaccinations are treated according to the same scheme as other adverse events from pharmaceuticals, moderated by the Pharmaceutical and Medical Device Agency (PMDA).

This paper analyzes some important features of current Japanese vaccination policy, which differs for each local government. Careful assessment of the Japanese vaccine approval policy landscape is important because there have been and will be drastic changes in vaccine policy in the next few years [5].

We consider the example of polio vaccination policy in Japan in order to illustrate a case which has overcome issues that became clear as the result of stakeholder analysis.

As seen in this history of a half-century, the NIP is vulnerable to public anxiety about vaccine safety. This paper analyzes some important features of the current Japanese vaccination policy, which differs for each local government. In our study we identified the critical turning point in NIP history and tried to evaluate the policy for influencing public climate regarding vaccine safety.

\section{Material and Methods}

\section{Study design}

This study is a systematic analysis of witnesses who represent key medical opinion leaders regarding the overall situation of the Japanese NIP and its application to one case of AEFI that resulted in a significant policy change.

\section{Data sources}

Several medical opinion leaders were addressed for semi-structured in-depth interviews. They were chosen to represent four stakeholder categories: Political layer, Policy makers, Practitioners, and the General Public. Interviewees and their attributions are listed in Table 2. These key informant interviews with health care professionals and policy makers were carried out from the local level to the national level. Participants were purposely selected according to a pub med data base literature search using key words including "vaccination" and we selected those who came up with insight on the topic. The study was conducted according to the ethical principles essential for research with key informant interviews. All the interviewees were informed about the aims of the interviews and the study he or she would be part of and were given the choice whether to participate or not. We asked questions regarding the challenges of Japanese public health system that had potentially undermined Japanese NIP and exacerbated vaccine gap. In these interviews, we tried to determine aspects of the decision making process which were not explicitly written on the guidelines. Subjective perceptions among the participants were explored. Field visits to health centers, as well as visits to local clinics, were conducted. 
We reached 23 interviewees but three of them declined.10 healthcare professionals agreed to be interviewed. In addition, 10 key informants agreed to be interviewed; three parliament members, two government officers, two local health authorities, and three persons from the non-government sector, including two leaders from the patients' advocacy groups.

Overall, 20 interviews were conducted. Interview collection was the responsibility of the research team. It is acknowledged that one fourth of the participants were workers for the central government, and there were several local clinic doctors and members of patients' groups.

\begin{tabular}{|c|c|c|c|}
\hline Layers & Key informant & Organization-field & Code \\
\hline \multirow[t]{3}{*}{ Political } & Parliament member & Opposition party & PM1 \\
\hline & Parliament member & Government party & PM2 \\
\hline & Parliament member & Opposition party & PM3 \\
\hline \multirow[t]{5}{*}{ Policy } & Government official & MHLW-vaccine & GO1 \\
\hline & Government official & MHLW-other & $\mathrm{GO} 2$ \\
\hline & Local health authority & $\begin{array}{l}\text { Institute-Tokyo wealthy } \\
\text { district }\end{array}$ & LA1 \\
\hline & Local health authority & $\begin{array}{l}\text { Institute-Tokyo poor } \\
\text { district }\end{array}$ & LA2 \\
\hline & $\begin{array}{l}\text { Vaccine } \quad \text { committee } \\
\text { member }\end{array}$ & $\begin{array}{l}\text { External member for } \\
\text { MHLW }\end{array}$ & $\mathrm{CM}$ \\
\hline \multirow[t]{10}{*}{ Practice } & Professor & $\begin{array}{l}\text { Public university-Internal } \\
\text { Medicine }\end{array}$ & P01 \\
\hline & Professor & $\begin{array}{l}\text { Public university-Health } \\
\text { policy }\end{array}$ & PO2 \\
\hline & Professor & $\begin{array}{l}\text { Public university- } \\
\text { Infectious disease }\end{array}$ & PO3 \\
\hline & Professor & $\begin{array}{l}\text { Private university- } \\
\text { Pediatrics }\end{array}$ & PO4 \\
\hline & $\begin{array}{l}\text { Physician working at } \\
\text { general hospital }\end{array}$ & US board certified doctor & CL1 \\
\hline & $\begin{array}{l}\text { Physician working at } \\
\text { general hospital }\end{array}$ & International hospital & CL2 \\
\hline & Nurse at general hospital & US board certified nurse & CL3 \\
\hline & Physician working at clinic & Obstetrician, urban area & CD1 \\
\hline & Physician working at clinic & Pediatrician, urban area & CD2 \\
\hline & Physician working at clinic & Pediatrician, rural area & CD3 \\
\hline \multirow[t]{2}{*}{ Public } & Patient group leader & Cervical cancer group & PG1 \\
\hline & Patient group leader & Polio group & PG2 \\
\hline
\end{tabular}

Table 2: List of interviewees.

\section{Evaluation and analysis}

We developed a discrete model of relevant modules to analyze the vaccine gap. The process of introducing new vaccines was dissected into five steps: production, licensure, routine scheduling, implementation and assurance [7]. We qualitatively analyzed interviewees' statements and categorized them in this diagram. This paper presents an idiographic representation of the views and concerns of the health care professionals who were interviewed. The analysis was conducted to articulate the diversity of opinions held by interviewees regarding the challenges of NIP. A potential proposal for solution was summarized on the basis of the qualitative analysis.

\section{Case study: polio vaccine switching}

After reviewing historic events, we focused on the political dynamisms of switching of Oral Polio Vaccine (OPV) into Inactivated Polio Vaccine (IPV) as a successful policy change.

\section{Results}

\section{Stakeholder analysis and summary proposals}

The interviewees commonly expressed their impression that Japanese vaccination policy is far behind that of other developed nations (GO1, 2, PM1, 2, PO1-4). Japanese policy makers are fast in reacting to AEFI cases; however, they are very slow in introducing new vaccines (CL1, CD1). Though the health of people is the highest priority, political and economic elements seem to be delaying the approval process. Among the parliament members we interviewed, selected from the three major parties, all of them stated that Japanese vaccination policy has been one of "national isolation" for the last 10 years (PM1-3). Opinion leaders pointed out the various challenges throughout the steps of vaccine introduction. Table 3 categorizes the modules of functions and related stakeholders in the logical step format. Different layers of opinion leaders mentioned different stakeholders as the cause of the Japanese vaccine gap. Table 4 shows the problems of segment identified by stakeholder layers of opinion leaders. The political layer attributed Japanese vaccine gap to the policy makers at the government. Government officials in charge of policy making, in contrast, attributed the issues to all three other stakeholder layers than themselves. Practitioners also identied problems out of the three layers except for themselves. General public appeared in favor of the political layer, pointing out the barriers between policy makers and practitioners. Their attitudes and proposed solutions were compiled into the following possible proposals.

\section{Evidence-based policy for routine vaccination}

The decision making process for introducing new routine vaccination has not been clear (CM). Expansion of voluntary vaccination by approving newly developed vaccine simply expands the autonomy for parents to vaccinate their children. In this environment, where group vaccination at school is not available, the vaccination rate is likely to decrease along with the increased autonomy of parents (LA1). However, failure to include new vaccines into routine vaccination does not only mean the lack of financial coverage, but also it jeopardizes the vaccines leading to lesser relief coverage for AEFI (PM3). Experts identified three key causes; human resources, cost effectiveness analysis, and risk management (GO1-CD3).

Human resources: Policy makers have attributed the "vaccine gap" to the lack of human resources of MHLW and PMDA. More medical experts are needed to accelerate the evaluation and approval process for newly developed vaccinations (PM1, PM2).

While policy making staff in the government complain that they have to draft policy with limited information, under the pressure of 
the "Big Voice" and arbitrary influence of political layers, they may be vulnerable to lobbying by the mega pharmaceutical companies (CL1). Developing a Japanese counterpart of the Advisory Committee on
Immunization Practice (ACIP), which has strict rules against obtaining stocks and receiving donations, is necessary (CL2).

\begin{tabular}{|c|c|c|c|c|}
\hline Steps & Modules & Key stakeholders & Area of science & Key approaches for solution \\
\hline Production & Research and development & $\begin{array}{l}\text { Academia, research institutions } \\
\text { and manufacturers }\end{array}$ & Biology and vaccinology & $\begin{array}{l}\text { Basic research, push and pull for } \\
\text { vaccine industries and scientists }\end{array}$ \\
\hline Licensure & Pharmaceutical approval & $\begin{array}{l}\text { Manufacturers and } \\
\text { (regulatory authorities) }\end{array}$ & Regulatory Science & Epidemiology, clinical trials \\
\hline Routine scheduling & $\begin{array}{l}\text { Funding, recommendation } \\
\text { and compensation programs }\end{array}$ & National diet and MHLW & Politics & $\begin{array}{l}\text { Lobbying, legitimacy, ethics, } \\
\text { fairness }\end{array}$ \\
\hline Implementation & Vaccination provision & $\begin{array}{l}\text { Municipalities and medical } \\
\text { professionals }\end{array}$ & Logistics & $\begin{array}{l}\text { Registry of vaccination history, } \\
\text { simultaneous inoculation, mult- } \\
\text { vaccine package }\end{array}$ \\
\hline Assurance & $\begin{array}{lr}\text { Risk management, } & \text { public } \\
\text { acceptance, } & \text { relief } \\
\text { compensation provision } & \end{array}$ & $\begin{array}{l}\text { Anti-vaccine activists, patient } \\
\text { groups, mass and social media, } \\
\text { MHLW }\end{array}$ & $\begin{array}{l}\text { Surveillance, education, } \\
\text { communication }\end{array}$ & $\begin{array}{l}\text { Causal investigation and } \\
\text { compensation of AEFI, Social } \\
\text { norm, risk communication strategy, } \\
\text { educational materials }\end{array}$ \\
\hline
\end{tabular}

Table 3: Logical steps and stakeholders for vaccine access improvement.

\begin{tabular}{|l|l|l|l|l|}
\hline \multirow{2}{*}{$\begin{array}{l}\text { Layers of interviewees who } \\
\text { identified the problems }\end{array}$} & \multicolumn{4}{|l|}{ Layers with problems causing vaccine gap } \\
\cline { 2 - 5 } & Political & Policy & Practice & Public \\
\hline Political & & $\mathrm{x}$ & & \\
\hline Policy & $\mathrm{x}$ & & $\mathrm{x}$ & $\mathrm{x}$ \\
\hline Practice & $\mathrm{x}$ & $\mathrm{x}$ & & $\mathrm{x}$ \\
\hline Public & & $\mathrm{x}$ & $\mathrm{x}$ & \\
\hline
\end{tabular}

schedule, then compensation programs for voluntary vaccines in case of AEFI should be improved (CD3). At the same time, more information concerning possible AEFI and the necessity of vaccination should be provided by the government. In addition, recipients should be encouraged to receive vaccines from the family doctors they have known for years, in order to avoid miscommunication and possible AEFI (CD2).

\section{Education for public}

Public perception regarding vaccination is generally negative, and

Table 4: Identified challenges of vaccine gap causes according to the segment of opinion leaders.

Cost effectiveness analysis: In the US, large expense is made on medical treatment, therefore it is rational to put effort in prevention (CL3). In the UK, the National Institute for Health and Care Excellence (NICE) conducts cost effectiveness analysis and its national health insurance system offers managed care system that prevents patients from having free access to experts. However, in Japan health technology assessment (HTA) has not been necessary for including new vaccines into routine schedule (CL1).

Risk management and surveillance: In Japan, those vaccinated are not tracked even in routine programs, and follow-up survey for vaccination history is not possible (GO1).

Besides, due to the past AEFI law suit cases, the government has become very sensitive and cautious (GO2). The government should make policy decisions not on the basis of AEFI-related media reports, but on science-based information (PO2). The government's actions have sometimes been taken without sufficient consideration. People lack the sense that some AEFI are something that is unavoidable (PM2). The Japanese government has reacted to this attitude by avoiding inclusion of new vaccines into routine schedule, which requires intense compensation scheme. Instead, it has increased the number of non-mandatory vaccines only, so that the government does not have to give a large amount of compensation in the case of AEFI (CD1). If it is an unrealistic option to include all vaccines into routine

the mass media's contribution to this negative perception is relatively large, such that it sometimes affects the national vaccination policy (GO2). There are no classes or teaching materials devoted specially to vaccinations for doctors at medical colleges, and none for the public. Education based on the notion of public health and preventive medicine is necessary (PO4).

The Internet offers mainly distorted information and it is accessed unequally by various groups in society. If based on an old education and the internet information, the vaccination policies can lead to discrimination (CL3). The government and pharmaceutical companies should provide more educational information (PG1). Though the voice of anti-vaccination group is big and it is often taken seriously as that of scientists, vaccination has a mission to protect vulnerable people from infection. Scientific evidence should be provided to antivaccination groups (CL1). Until recently, vaccination has never been a main topic either among stakeholders or other groups. Medical workers should pay more attention to vaccines (PG2).

\section{Domestic vaccine manufacturers}

Experts have also pointed out the vulnerability of Japanese manufacturers in their research, development and production of vaccines. The major causes of this problem are said to be the following three factors:

Scale of manufacturers: Japanese domestic vaccine manufacturers have remained smaller in business scale, in comparison to the overseas vaccine-producing pharmaceutical companies. Most foreign 
manufacturers are parts of the global mega-pharma industry, while Japanese manufacturers are often based on research laboratories. Thus the domestic manufacturers have limited financial backbone to invest in the research and development of new vaccines (PO1, PO2, CL3).

Vaccine hesitancy: The nationwide anti-vaccine movement of the 1990s has demotivated Japanese large pharmaceutical companies from retaining their vaccine divisions, considering the risk of lawsuit and the large amount of compensation. At the same time, the drastically decreased vaccination rate of flu vaccines devastated the vaccine business and its related resources. Scarce human resources and research infrastructures have prevented Japan from developing new vaccines over the past quarter century (GO2, CM).

Industry protection policy: Japanese government protected domestic manufacturers by the regulations and public subsidies, which might have spoiled the domestic company in their international competitiveness (CL1, CM).

\section{Case study: polio vaccine switching}

We examined a case illustrating the national conservative policy and its recent rapid change into evidence-based policy induced by one local government's initiative which caught media attention and influenced public perception towards safety of IPV against OPV.

Japan had a polio outbreak in 1959. The government initiated an emergency polio protocol and polio vaccination became mandatory in 1961. At that time, Japan had no domestic production capacity. The government urgently imported foreign OPV, followed by the licensed domestic production. In 1964, routine vaccination of domestic OPV immunization started and eliminated wild-polio in 1980. The government continued using OPV, even though it caused some cases of vaccine-associated paralytic poliomyelitis (VAPP). From 2001 to 2010, in 10 years, 15 people (1.4 per 10 million) developed VAPP after OPV vaccination and 6 people got secondary infection [8]. The Polio Research Center once applied for IPV production approval in 2001 but dropped its application for IPV in 2005. Four domestic manufacturers started development of quarto-valent Diphtheria, Pertussis, Tetanus (DPT)-IPV since 2002. Despite the recognition of VAPP burden overweighing the wild polio, and inclusion of VAPP into national surveillance of infectious diseases in 2004, it was not until in 2010 that MHLW's national immunization committee decided to introduce IPV in Japan and the development of IPV by non-Japanese producers was initiated [8]. MHLW also urged domestic manufacturers to accelerate DPT-IPV production in 2010. In August 2011, at a meeting of the committee for smooth transition to IPV, they concluded that "MHLW should be trying hard to introduce IPV; meanwhile, the use of OPV cannot be terminated [9]." From October 2011, individual imports of IPV started to increase and about $3.4 \%$ and $0.6 \%$ of clinics and hospitals were offering IPV [10]. OPV users dropped by $17.5 \%$ in 2011, compared to 2010 [10].

OPV recipients in Kanagawa prefecture, which made a decision to import IPV at the local level, dropped by $21.5 \%$ in April-June 2011, compared to the previous year. The Kanagawa government has set its own guideline for severe AEFI, which is similar to that of the relief system for victims of adverse reactions. The cost of vaccination had to be covered by recipients, as no subsidy is allocated by the government [11]. This policy of Kanagawa has drawn significant media attention and it has led to a rapid shift from OPV to IPV in Japan. IPV was introduced in September 2012 as a routine vaccination with full compensation.
The case of polio shows the current improvement in Japanese vaccination policy. The challenges that have kept the Japanese government using OPV are related to the problems identified in the previous section: lack of evidence-based policy making and weak manufacturing capacities. The changing of the balance of prevention vs. AEFI between OPV and IPV were not reflected in policy changes made by government officials. There had been only one domestic manufacturer of polio vaccine, which was only OPV. It is also said that the government tried to use domestic manufacturers which were not capable of producing IPVs. However, the public paid attention to the safety of OPV and learned that IPV is a better option in Japan. This led to the drop in vaccination rate of OPV. In spite of the delayed government decision, there was one local government that independently made a political decision to import IPV for its local residents. This decision was largely welcomed by the public, who were concerned about OPV safety, and this amplified the broader public attention. The central government later had to switch to IPV in response to the public climate. This is a good example of the evidence policy leading to changes through public awareness and education.

\section{Discussion}

The root causes of the current vaccine gap have to do with the lack of understanding among the public regarding the aims of vaccination. The first aim is to enhance total population immunity, to eliminate Vaccine Preventable Diseases (VPD) and to protect individuals from VPD. However, people by and large believe that routine vaccination for some diseases is a necessary obligation for child health care, and that voluntary vaccination is totally an individual option. This perception is shared in a Canadian study that showed people's belief that if a vaccine will benefit them individually, it will be provided to them at no cost [12]. Canadian study contends that private access to approved but unfunded vaccines should be reconsidered and encouraged since the number of "approved but unfunded vaccines" is likely to grow [12]. In addition, it shows that professional organizations can play a key role, since the public generally values expert advice that is independent from the government and industry. The result of our study is in line with this Canadian study. In terms of biosecurity, maintaining domestic vaccine production could be an important aim. As for future policy, evidence-based policy, appropriate risk monitoring, and a surveillance system should be established. For this, an ACIP-style organization, whose members have no ties to government or pharmaceutical companies, needs to be established. This is in line with the previous research of Saitoh et al. [2] which argued for the necessity of establishing an effective National Immunization Technical Advisory Group (NITAG).

Regarding education and communication, medical professionals should reach out to the public, there should be teaching methods available to medical professionals, and science-based neutral information should be provided to the public by the medical professionals or government. As in Japan, in Holland, the information that people search for the most is about adverse reactions. Lehman et al. showed that social media have more critically evaluated vaccination information than have the news media [13] and that Internet searches on the word "vaccination" showed more anti-vaccination websites than pro-vaccination ones [14]. Despite the massive political influence of anti-vaccine activists, the majority of under-vaccination is said to be associated with the "forgetting" factor that happens due to the complexity of the schedule. This is mentioned in previous research overseas done by Smith et al. [15]. Another Dutch research paper 
concluded that in the Netherlands, parents receive oral information about the NIP at a nurse's home visit, that parents perceived health care workers as the most reliable source of vaccine-related information, and that the information provided by the NIP might be sufficient for most parents [16]. This oral information system might be an option in Japan. The measures such as improved notification and record-taking, group vaccination in the municipalities, guiding materials for practitioners and public, simultaneous inoculation by MHLW should be taken. Research in Brazil shows that easy access to such things as temporary vaccination points and public-private partnerships has led to good control over VPD [17]. Since Japan has a higher rate of VPD compared to other developed nations [18], Japan could follow Brazil's case and restart group vaccination. However, our evidence is still small; therefore, further research on cost effectiveness analysis is necessary.

In response to the problems that experts have pointed out, there are some possible solutions to improve the situations.

We need more time, finance, and human resources to improve cost effectiveness analysis capacities in Japan. For the short term, we should refer to the overseas studies for upfront decision making. Meanwhile, the government should invest in cultivating professionals in epidemiology and health economics. Currently, the schools of public health are underdeveloped. There are only six public health schools in Japan, and most of them are parts of medical schools. This fact indicates that public health is not recognized as an independent area of medical science.

Risk assessment and management can be systematically improved through collective registry of individual vaccination history and collective surveillance of AEFI. Currently, AEFIs are collected through both doctors and manufacturers, but risks are not assessed in appropriate study designs, such as by use of case control studies. Most municipalities retain records of the children to whom they have provided routine vaccinations, but if the children move elsewhere, it may disrupt tracking. Maternal and childcare notebooks keep vaccination histories but they have not been integrated into a nationwide registry system. A social security numbering system could provide an infrastructure for AEFI risk assessment. So as to avoid insufficient compensation and thereby to exaggerate vaccine hesitancy, more adequate AEFI compensation, including voluntary vaccination, could be provided through the collection and pooling of a surcharge fee upon vaccination.

Japan has already experienced an excellent example of raised vaccine awareness in the polio case. It was an interactive process. The grassroots level anxiety about OPV safety was captured by the political leadership. Then the prefectural decision amplified nation-wide concern and triggered a public momentum to request the government to switch from OPV to IPV. It should be noted that the entire process was backed up with correct scientific evidence. Since it is sometimes difficult for the national government to lead policy change, local leadership or public climate is sometimes better able to facilitate the adaptation of evidence-based policy making.

The polio case also illustrated the fact that excess domestic production may have partly caused the vaccine gap. It is generally true that the government should purely focus on improving public health, regardless of the industry's interest. Therefore, so as long as it is needed, governments are expected to approve and include overseas vaccines into the NIP. The MHLW decided to include into routine schedule the following: Hib, PCV and HPV in April 2013, and varicella and adult PPV in October 2014. However, retention of domestic vaccine production capacity is essential for national health security. In the case of the pandemic of flu in 2009, the Ministry of Health, Labour and Welfare decided to import H1N1 vaccine from overseas manufacturers. This shows that vaccines play a critical role in protecting a nation under health emergencies. In the situation of emerging diseases, global logistics may not sufficiently work to deliver necessary medical countermeasures. Thus, a nation should maintain essential capacities to manufacture health protection resources, such as vaccines, as a part of national security policy.

This study has some limitations. This study does not test the causality of the problems identified in a context reaching beyond the opinions of the experts who responded to our interviews based on their experiences. The proposed solutions can also be questioned with respect to their plans for implementation and expected outcomes. The value of this study lies in its consolidation of the opinions of professionals into a structured framework, thereby offering public health specialists a basis to develop their own policy proposals.

\section{Conclusions}

Although the national government of Japan has improved the vaccine gap recently, there remains the systematic problems in area of public health human resources, surveillance and risk management system, and industrial capacities. Polio case showed that political leadership at the local level could be an efficient strategy to introduce scientific evidence into public policy at national level.

\section{References}

1. Tuberculosis and infectious diseases control division, Health bureau, Ministry of Health, Labour and Welfare (2014) National Immunization Program.

2. Saitoh A, Okabe N (2012) Current issues with the immunization program in Japan: Can we fill the "vaccine gap"? Vaccine 30: 4752-4756.

3. Nakatani H, Sano T, Iuchi T (2002) Development of Vaccination Policy in Japan: Current Issues and Policy Directions. Jpn J Infec Dis 55: 101-111.

4. Vaccine policy committee (2013) Reconsidering of National Immunization Program.

5. Health safety research center, Trend of new vaccinations. Kawasaki city bureau.

6. Asahi newspaper editorial team (1996) Risk of infection from HIVcontaminated blood product recognized in 1983. National policy switch. Asahi newspaper.

7. Yobousesshu Bukai, MHLW (2004) Policy briefing for 2nd hand infection of OPV.

8. Yobosesshu Bukai, MHLW (2010) 6th meeting minutes.

9. Yobousesshu Bukai, MHLW (2011) 1st conference for smooth implementation of IPV minutes.

10. Kanagawa prefecture hospital's association (2011) Inactivated polio vaccination.

11. http://www.pref.kanagawa.jp/cnt/f360694/

12. Scheifele D, Ward B, Halperin S, McNeil S, Crowcroft N, et al. (2014) Approved but non-funded vaccines: Accessing individual protection. Vaccine 32: 766-770.

13. Lehman BA, Ruiter RA, Kok G (2013) A qualitative study of the coverage of influenza vaccination on Dutch news sites and social media websites. BMC Public Health 13: 547.

14. Wolfe RM, Sharp LK (2005) Vaccination or immunization? The impact of search terms on the internet. J Health Commun 10: 537-551. 
Citation: Tomisaka M, Makino T, Marui E (2015) Overcoming the Japanese "Vaccine Gap": An Analysis of Medical Leaders' Witness. J Vaccines Vaccin 6: 263. doi:10.4172/2157-7560.1000263

Page 7 of 7

15. Smith P, Chu S, Baker L (2004) Children who have received no vaccines: Who are they and where do they live? Pediatrics 114: 187-195.

16. Harmsen I, Doorman G, Mollema L, Ruiter R, Kok G, et al. (2013)

Parental information-seeking behavior in childhood vaccinations. BMC Public Health 13: 1219.
17. Barreto M, Teixeira M, Bastos F, Timens A, Barata R, et al. (2011) Successes and failures in the control of infectious diseases in Brazil: social and environmental context, policies, interventions and research needs. Lancet 377: 1877-1889.

18. Association of biological manufacturers in Japan (2011) Report of VPD. 